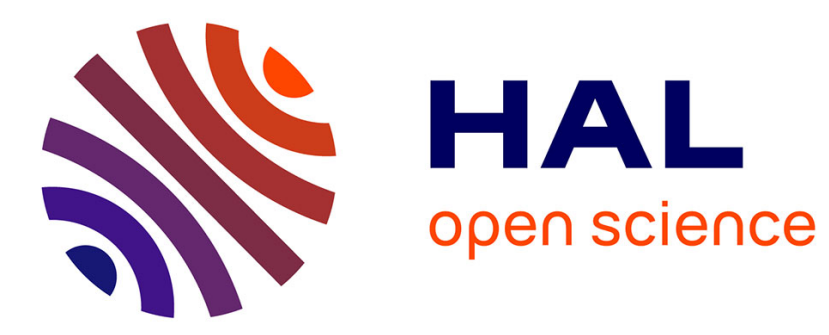

\title{
Chemical Functionalization of Nanodiamonds: Opportunities and Challenges Ahead
} Giacomo Reina, Li Zhao, Alberto Bianco, Naoki Komatsu

\section{To cite this version:}

Giacomo Reina, Li Zhao, Alberto Bianco, Naoki Komatsu. Chemical Functionalization of Nanodiamonds: Opportunities and Challenges Ahead. Angewandte Chemie International Edition, 2019, 58 (50), pp.17918-17929. 10.1002/anie.201905997 . hal-02994625

\section{HAL Id: hal-02994625 \\ https://hal.science/hal-02994625}

Submitted on 12 Nov 2020

HAL is a multi-disciplinary open access archive for the deposit and dissemination of scientific research documents, whether they are published or not. The documents may come from teaching and research institutions in France or abroad, or from public or private research centers.
L'archive ouverte pluridisciplinaire HAL, est destinée au dépôt et à la diffusion de documents scientifiques de niveau recherche, publiés ou non, émanant des établissements d'enseignement et de recherche français ou étrangers, des laboratoires publics ou privés. 


\title{
Chemical functionalization of nanodiamonds: opportunities and challenges ahead
}

\author{
Giacomo Reina, ${ }^{a}$ Li Zhao, ${ }^{b, c}$ Alberto Bianco, ${ }^{a, b *}$ Naoki Komatsu ${ }^{b *}$ \\ a University of Strasbourg, CNRS, Immunology, Immunopathology and Therapeutic \\ Chemistry, UPR 3572, 67000 Strasbourg, France \\ ${ }^{b}$ Graduate School of Human and Environmental Studies, Kyoto University, Sakyo-ku, \\ Kyoto 606-8501, Japan \\ c State Key Laboratory of Radiation Medicine and Protection, School of Radiation \\ Medicine and Protection \& School for Radiological and Interdisciplinary Sciences (RAD- \\ X), Collaborative Innovation Center of Radiation Medicine of Jiangsu Higher Education \\ Institutions, Soochow University, Suzhou, Jiangsu 215123, China
}

Corresponding author:komatsu.naoki.7w@kyoto-u.ac.jp; a.bianco@ibmc-cnrs.unistra.fr

Dedicated to Prof. Gianfranco Scorrano on the occasion of his $80^{\text {th }}$ birthday and to Prof. Takahide Kimura for his 70th birthday

Keywords carbon materials; diamond; functionalisation; therapy; imaging

Dedicated to Prof. Gianfranco Scorrano on the occasion of his 80th birthday and to Prof. Takahide Kimura for his 70th birthday 


\begin{abstract}
Nanodiamond (ND)-based technologies are flourishing in a wide variety of fields spanning from electronics and optics to biomedicine. NDs show interesting physicochemical properties such as hardness, stiffness and chemical stability. Additionally, NDs can undergo ad hoc core and surface functionalization, which tailors them for the desired applications. Noteworthy, the properties of NDs together with their surface chemistry are highly dependent on the synthetic method used to prepare them. In this minireview, we critically describe the preparation of NDs from a viewpoint of materials chemistry. The different methodologies of synthesis, purification, surface functionalization and the biomedical applications are thoroughly and critically discussed. New synthetic approaches as well as limits and obstacles of NDs are presented and analyzed. This minireview is particularly addressed to chemists who have started or are willing to explore the ND science and technology.
\end{abstract}




\section{Introduction}

Nowadays, nanotechnology is widening its application field and it is integrated more and more in our daily life. The preparation of pure, homogeneous and monodisperse nanoobjects is highly desirable. In this context, nanodiamods (NDs) have been extensively studied finding wide and transversal cutting-edge applications in several domains. ${ }^{[1-3]}$ NDs are a family of nanomaterials that shares a core constituted by a common $\mathrm{sp}^{3}$ carbon structure, but differs in size, shape and surface chemistry. By different synthetic approaches it is possible to obtain very small particles of 3 to $4 \mathrm{~nm}$ or homogeneous films with micron size thickness. The preparation methods also influence the quality, the quantity and surface chemistry of the final material. The most popular synthetic strategies for the production of NDs comprise detonation (D-NDs), high pressure-high temperature (HPHT-NDs) and chemical vapor deposition (CVD-NDs). D-NDs are monodisperse diamond particles produced in large scale by exploding appropriate precursors, with a relatively small crystalline size. HPHT-NDs are obtained from a top-down process by milling HPHT diamond followed by levigation. CVD-NDs can be generated on different substrates leading to the formation of films. As-prepared NDs share the same core structure imparting mechanical and optical properties similar to bulk diamond. In particular, NDs are characterized by high Young's modulus, high stiffness, excellent thermal conductivity, high refractive index and stability in harsh conditions. Together with the features of the core, NDs are endowed with a versatile surface chemistry, which allows to expand their use in different fields. Playing with the functional groups that are present or can be added onto the surface of NDs, we can tune both their macroscopic (e.g. hydrophilicity and colloidal stability) and microscopic (e.g. reactivity) properties making the prepared NDs suitable for the desired applications. Additional treatments can be performed to modify the $\mathrm{sp}^{3}$ core. In this case, high energy ions are used to implant defects inside the ND lattice (doping). ${ }^{[4]}$ These defects incorporated into the $\mathrm{sp}^{3}$ structure, called color centers, correspond to new atoms or vacancies into the lattice and produce fluorescence NDs, making them suitable for electronics and bioimaging. Currently, different ND technologies in lubricant and polymer science have already reached late stage trial and few of them are commercially available. ${ }^{[5]}$ However, in some other fields such as biomedicine, several challenges still need to be faced before a safe application 
of ND-based technologies reaches the market. Moreover, there is still room for improvement regarding purification and functionalization protocols. For instance, D-NDs require extensive post-synthesis modifications to obtain stable dispersions, while doping generally needs high temperature, being incompatible with most of the surface treatments. For these reasons, different multi-functionalization protocols have been recently explored, proving how crucial is tailoring the synthetic and functionalization strategies to expand ND applications. In this minireview we will critically focus on the preparation and chemical functionalization of NDs, particularly foreseeing their applications into the biological and biomedical fields.

\section{Methods of synthesis: HPHT and detonation}

NDs can be produced by various top-down and bottom-up methodologies. ${ }^{[6]}$ Bottom-up methods build the carbon lattice from molecular precursors including explosives for DNDs or small hydrocarbons for CVD-NDs. Alternatively, top-down protocols are based on the controlled fragmentation of micron sized diamonds by use of milling or ultrasound. ${ }^{[6]}$ Since the most exploited synthetic protocols are HPHT and detonation, we decided to describe the details of the processes to obtain these types of NDs.

D-NDs are produced through explosion of a mixture of 2,4,6-trinitrotoluene (TNT) and 1,3,5-trinitroperhydro-1,3,5-triazine (hexogen). During this process, nanodroplets of liquid carbon are formed and converted to D-NDs after fast cooling. The detonation soot is then subjected to intense oxidative purification steps to remove iron impurity and $\mathrm{sp}^{2}$ carbons and extract the desired D-NDs. The detonation process allows the production of homogeneous particles with a very narrow size distribution, typically 1 to $10 \mathrm{~nm}$.

In the case of HPHT, single crystalline diamonds can be obtained from graphite or other carbon sources such as carbon nanotubes ${ }^{[7]}$ or fullerenes ${ }^{[8]}$ at high temperature and high pressure. These conditions cause thermodynamic transition of the carbon phase from $\mathrm{sp}^{2}$ to $\mathrm{sp}^{3}$ hybridization. HPHT process is quite versatile, as pressure and temperature can be finely tuned to control the size of the prepared bulk diamond. [9] To obtain NDs of desired size, as-produced bulk diamonds must then undergo a fragmentation process via bead-assisted sonic disintegration or mechanical ball milling, giving high quality NDs typically of 20-30 nm up to few microns. ${ }^{\left[{ }^{[9]}\right.}$ Alternatively, an interesting crushing method 
reported the use of a hydraulic press in the presence of $\mathrm{NaF}$ salts yielding nanoparticles with size below $20 \mathrm{~nm} \cdot{ }^{[10]}$ Subsequently, this type of NDs can be subjected to ionimplantation followed by high temperature annealing to generate nitrogen vacancy $(\mathrm{N}-\mathrm{V})$ centers for endowing NDs with fluorescence properties. ${ }^{[11]}$

If we compare HPHT-NDs and D-NDs, the shape, the interparticle interactions and the surface differ visibly (Fig. 1).

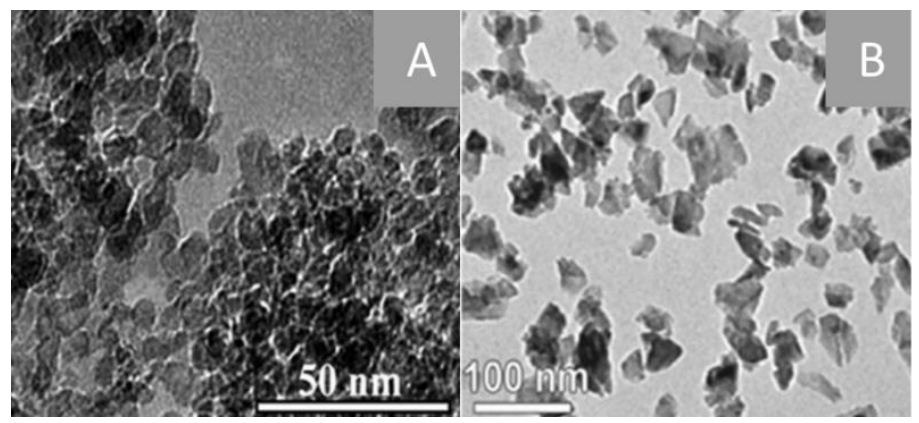

Figure 1. TEM images of D-NDs (A) and HPHT-NDs (B). Reprinted with permission from ref. [3]. Copyright 2017 Elsevier.

In the case of HPHT, the size of the final particles is tuned by the milling conditions of the bulk diamonds. ${ }^{[12]}$ These NDs bear lower quantity of $\mathrm{sp}^{2}$ carbon compared to D-NDs. ${ }^{[12]}$ Moreover, the fragmentation process allows to obtain sharp faced materials with a lower tendency to form agglomerates. ${ }^{[12]}$ On the other hand, detonation is a bottom-up technology where the primary monodisperse (narrow size distribution) ND particles are formed during the explosion. ${ }^{[13]}$ But, the produced soot is a complex mixture of amorphous, graphitic carbon with traces of the metal present in the detonation chamber, thus requiring an efficient purification protocol. ${ }^{[6]}$ Additionally, $\mathrm{sp}^{2}$ carbon impurities can easily remain into the cleaned D-NDs. D-NDs also shows a significant agglomeration tendency, due to their different surface potentials. ${ }^{[6]}$ Concerning surface functionalities, detonation process allows the introduction of a wide variety and quantity of functional groups rendering D-NDs more versatile for chemical modifications ${ }^{[6]}$ than HPHT-NDs where hydroxyls are the dominant functions. ${ }^{[12]}$ In addition, color centers can be generated more easily in HPHT-NDs than in D-NDs, because they can host more defects. ${ }^{[5]}$ 
Overall, HPHT- and D-NDs show high potentials and versatility allowing to expand their application in a wide variety of technological fields. However, we should choose the appropriate NDs by taking into consideration the characteristics of the particles and the technological needs.

\section{Post-synthetic treatments}

Prior to each utilization, NDs require an accurate purification, deagglomeration and fractionation process. The different steps are really crucial and strongly affect the final quality of the produced material. Ideally, the overall post-synthetic treatments should be efficient, cost effective, scalable, and reduce the batch to batch variability. Specific postsynthetic protocols have been extensively described for D-NDs and HPHT-NDs (Fig. 2).
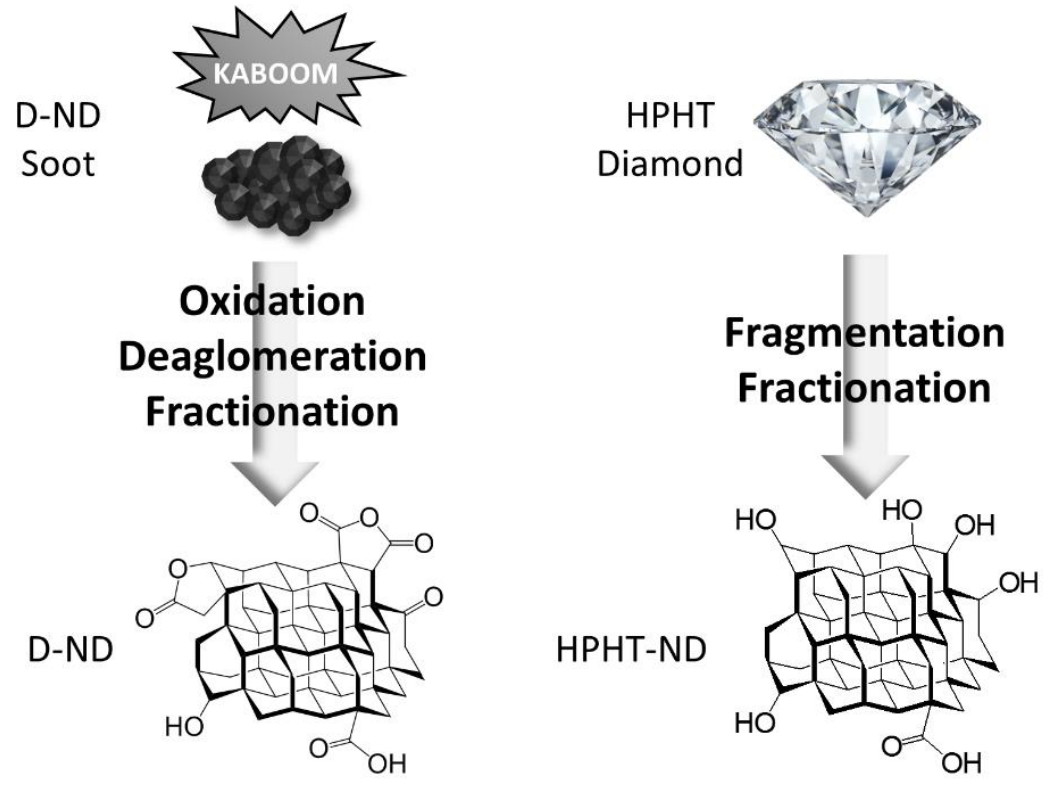

Figure 2. Illustration of the synthesis and post-synthetic treatments in the preparation of D-NDs and HPHT-NDs.

Besides, the soots produced via detonation contain the desired NDs, together with nondiamond carbon and residual metals which makes purification the most delicate protocol for D-NDs. On the other hand, the purification of HPHT-NDs is nearly not necessary as the crude product into the synthetic chamber is composed by almost pure diamond. ${ }^{[12]}$ 
Hence for HPHT-NDs, fractionation is the most important step to obtain a sharpen size distribution material.

Purification of the HPHT diamond is performed to remove the non-diamond carbon traces present on the surfaces. ${ }^{[14]}$ Instead, for D-NDs it is necessary to extract the diamond phase from the detonation soot. The major impurities present in the soot comprise metallic contaminants (1-8\%) and non-diamond carbon that can reach concentration of $80 \% .{ }^{[15]}$ Typically, acid treatments using $\mathrm{HCl}, \mathrm{HBr}$ or $\mathrm{HF}$ can remove both non-diamond carbon and metal impurities. ${ }^{[16]}$ More complex purification protocols foresee the use of different oxidant mixtures (e.g. $\mathrm{HCl} / \mathrm{HNO}_{3}, \mathrm{H}_{2} \mathrm{O}_{2} / \mathrm{NaOH}$ ). ${ }^{[16]}$ The use of these liquid phase protocols are not environmental friendly, hazardous and costly. ${ }^{[16]}$ For these reasons, new gaseous-phase treatments (e.g. ozone, hydrogen) are now into the developing stage. We would like to stress that in case HPHT-NDs, purification step is strongly advised after the doping process, where the high temperature necessary in the ion implantation and annealing may induce graphitization of the diamond surface. In addition, the oxidative/reductive purification protocols modulate the types of organic groups, which are essential to select the further surface functionalization.

After the purification steps, the D-NDs need to undergo deagglomeration. High energy ball milling has been reported as the best deagglomeration protocol..17] This technique induces sample contamination due to the inevitable breaking of the milling media (i.e. zirconia, stainless steel). Alternatively to classic milling, the use of sugar or salts has been studied. ${ }^{[18]}$ Due to the lower tendency to agglomerate, in the case of HPHT-NDs, milder de-agglomeration such as sonication can be performed. ${ }^{[14]}$

The last step of the post synthetic treatments is the fractionation. This step is important in the case of D-NDs to divide the different agglomerate size, while in the case of HPHTNDs it helps to select the desired size. Moreover, for D-NDs fractionation can be not necessary since narrow size agglomerates and well as single digit dispersions can be obtained by milling. ${ }^{[14]}$ In the case of HPHT-NDs, fractionation is the most delicate step due to a wide particle size distribution after crushing. The fractionation is generally performed via centrifugation, provided that the NDs have a good colloidal stability in the appropriate solvent. Additionally, ultracentrifugation and density gradient centrifugation 
can be applied. ${ }^{[14]}$ These processes can be performed more times to narrow the size distribution of the final product. We would like to underline that the yield of fractionation is highly dependent on the starting NDs. As HPHT-NDs are produced by a top-down approach, the collected fractions may be composed by particles with a wider size distribution.

\section{Surface functionalization}

In many applications, it is necessary that NDs may undergo specific surface functionalizations. The chemical modification of ND surface allows to tune the interaction of the nanoparticle with the surrounding media. This behavior is difficult to control. For instance, dispersibility is crucial for the preparation of homogeneous ND dispersions, while playing with organic functions we can tune the efficiency of NDs to adsorb ions or molecules (e.g. drugs in the case of biomedical applications). ${ }^{[6]}$ ND chemistry has been well-established. The NDs are characterized by a robust $\mathrm{sp}^{3}$ core structure that make them highly stable under strong acidic or basic conditions. On the other hand, high temperatures are able to induce surface graphitization leading to a loss of the functional groups ${ }^{[6]}$ HPHT-ND surface resemble that of bulk diamond, and it is characterized mainly by the presence of hydroxyl groups and few carboxylic groups. ${ }^{[12]}$ D-NDs are instead considered the carbon nanostructure with the highest variety of surface diverse functional groups. ${ }^{[12]}$ In particular, hydroxyl and carboxylic groups, epoxides and lactones have been identified onto the D-ND surface. On top of this abundance, we must consider that there could be a high variability between the different producers or even in the batches. In addition, to complicate the situation, the characterization and the quantification of the organic functions is rather difficult giving often inconclusive results. For these reasons, as a common procedure before any surface functionalization, the NDs are first submitted to a process of surface homogenization. Depending on the desired functionalities, the homogenization can be performed under oxidative or reductive conditions (Scheme 1). ${ }^{[5]}$ 


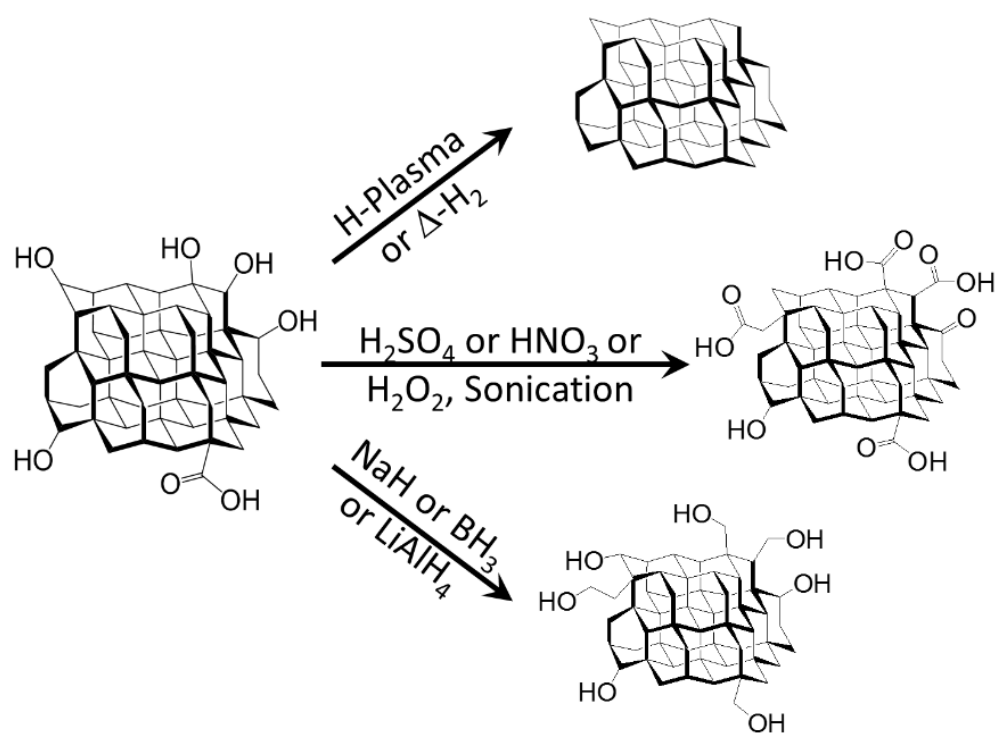

Scheme 1. Chemical reactions performed on NDs to reduce or oxidize their surface.

As presented above, NDs are characterized by the presence of oxygen-containing functional groups. Oxidative conditions, such as treatments with sulfuric acid, nitric acid or hydrogen peroxide under sonication, are able to enhance the amount of carboxylic acids on the surface of D-NDs or HPHT-NDs. ${ }^{[19]}$ NDs can be alternatively submitted to a reduction process using wet chemistry or plasma. ${ }^{[19]}$ Wet chemistry reduction can be performed using various common reductant such as hydrides (Scheme 1). ${ }^{[19]}$ The aim of this process is to enhance the presence of hydroxyl groups on top of the NDs. H-plasma can be also implemented to efficiently reduce ND surface. Depending on the conditions and the temperature, the reduction can enhance the formation of $\mathrm{OH}$ or lead to hydrogenterminated NDs. ${ }^{[13]}$ There is not a single protocol to obtain the homogenization of the total ND surface but there is rather an enrichment of the desired functional groups. Moreover, if on one side homogenization allows the uniformity of the ND surface, on the other, it depletes the presence of other organics that may be used for orthogonal functionalization. At the moment, no pretreatments have been reported aiming to control the presence of more than one organic function, making the NDs prone to multifunctionalization. We believe that there are a lot of possibilities for improvement in this direction. 
After surface homogenization, the NDs are subjected to further functionalization. The main strategies proposed for ND functionalization covers both covalent and non-covalent approaches. Non-covalent functionalization relies mainly on two types of interactions: electrostatic (Scheme 2) or hydrophobic (Scheme 3). ${ }^{[20]}$ For the first one, oxidized NDs are generally used, because their surface is highly negatively charged due to the presence of carboxylic groups (Scheme 2).

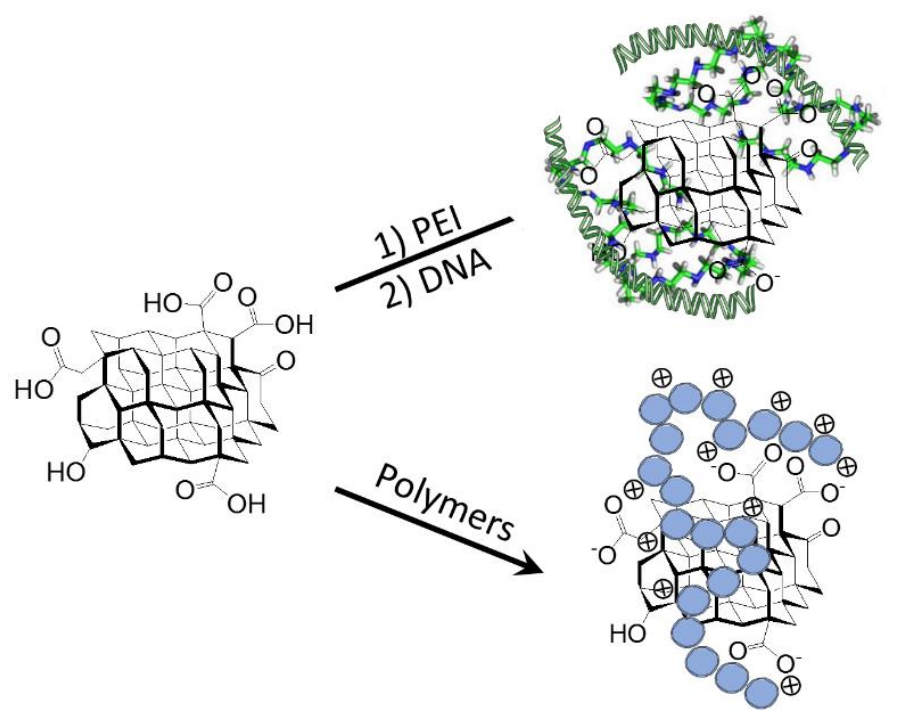

Scheme 2. Non-covalent functionalization of NDs through electrostatic interactions.

In the second case, the target molecule can be adsorbed onto ND surface via apolar interactions (Scheme 3). ${ }^{[12]}$ The non-covalent functionalization is simple and based on the self-assembly between the NDs and the desired molecule, and it has been extensively used for the preparation of composites and in drug delivery. ${ }^{[5,21]}$ 


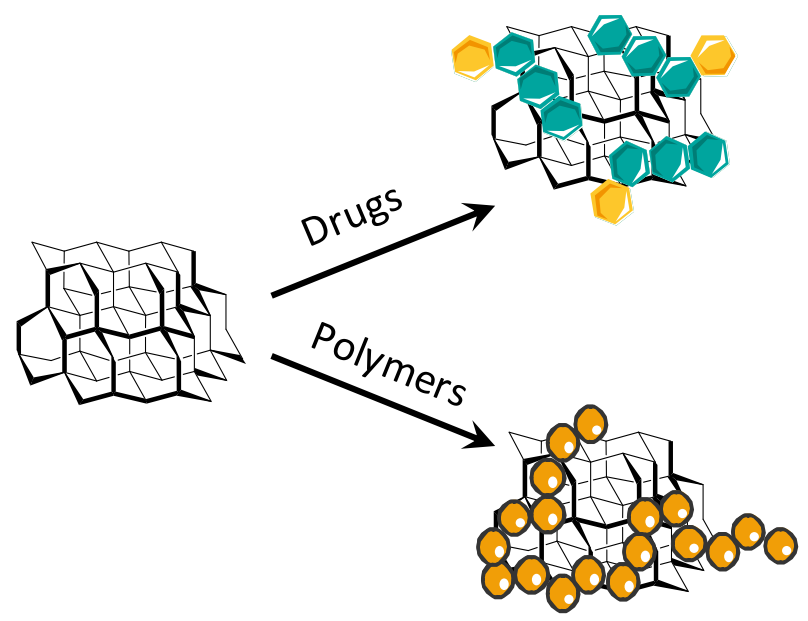

Scheme 3. Non-covalent functionalization of NDs through hydrophobic interactions.

For drug delivery applications, the drugs can be adsorbed on top of the ND surface and then released in the desired environment. ${ }^{[22,23]}$ However, non-covalent functionalization suffers from lack of reproducibility. This drawback is due to the scarce control of the overall adsorption process. Indeed, the inhomogeneous ND surface together with the non-uniform agglomeration state may dramatically affect the interaction between the particles and the adsorbed molecules.

Regarding the covalent approaches, the most exploited groups are the carboxylic and the hydroxyl groups. The carboxylic groups are quite abundant on D-NDs and can react with amines after activation. ${ }^{[23]}$ Efficient activation of the carboxylic groups is obtained by thionyl chloride able to convert the carboxylic acid into the acyl chloride (Scheme 4). 


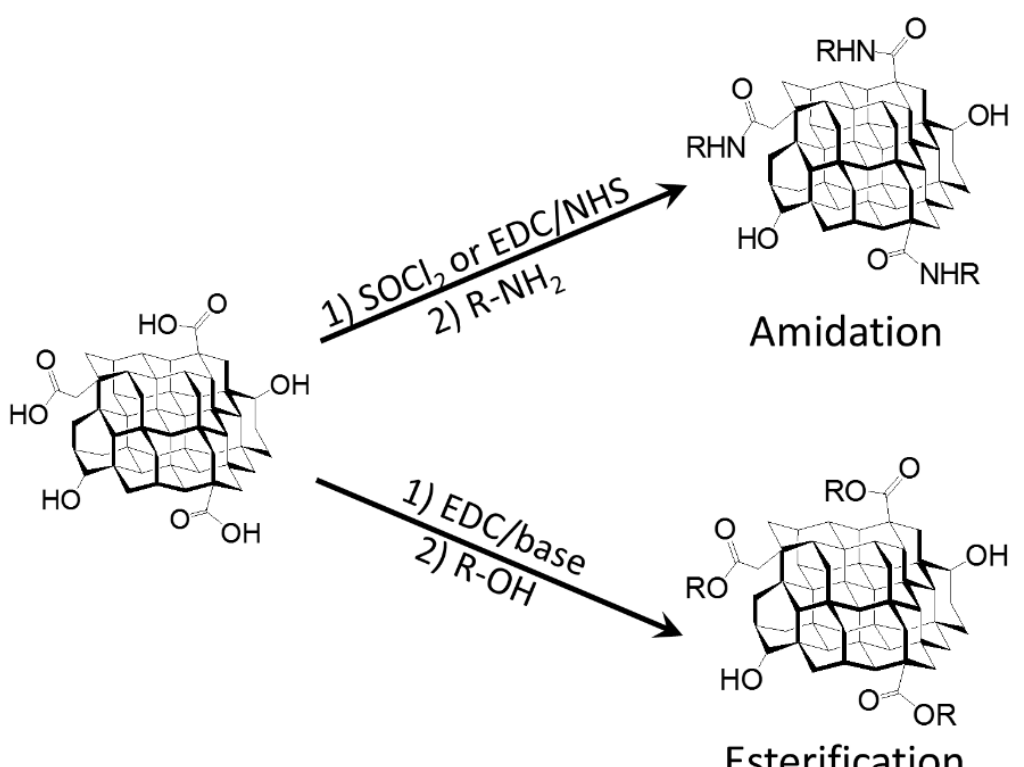

Scheme 4. Functionalization of the carboxylic functions of NDs $(R=$ proteins, peptides, dyes).

However, this reagent is not specific and can also convert hydroxyl groups to chlorides via nucleophilic substitution. ${ }^{[24]}$ Milder activation protocols have been reported using classical carbodiimide/succinimide activation (EDC/NHS) [1-ethyl-3-(3dimethylaminopropyl)carbodiimide/ $\mathrm{N}$-hydroxysuccinimide] that allows reactions in water or buffer at room temperature. ${ }^{[23]}$ This methodology has widened the applications of NDs to the biomedical field. Several studies showed efficient grafting of a large variety of moieties including small molecules, dyes, monomers, polymers or proteins (Scheme 4).[23] Another extensively exploited reaction targeting the carboxylic groups is the esterification. In this case, after activation using carbodiimides and appropriate bases, the NDs can be functionalized with the desired alcohol [e.g. polyethylene glycol (PEG) or

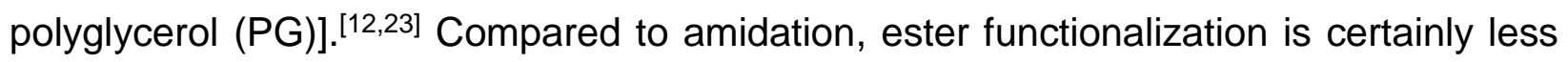
stable and more prone to hydrolysis. Esterification of NDs was normally used to graft biopolymers in order to enhance the colloidal stability and blood circulation. ${ }^{[25,26]}$

Another popular functionalization regards the derivatization of the hydroxyl groups. Such groups are naturally present in both HPHT-NDs and D-NDs and their quantity can be 
enhanced via an appropriate surface homogenization. Hydroxyl groups can be functionalized via esterification reaction in similar conditions described for $\mathrm{COOH}^{\left[{ }^{27]}\right]}$ Another attractive strategy for the derivatization of NDs is the reaction with siloxanes (Scheme 5). ${ }^{[12]}$

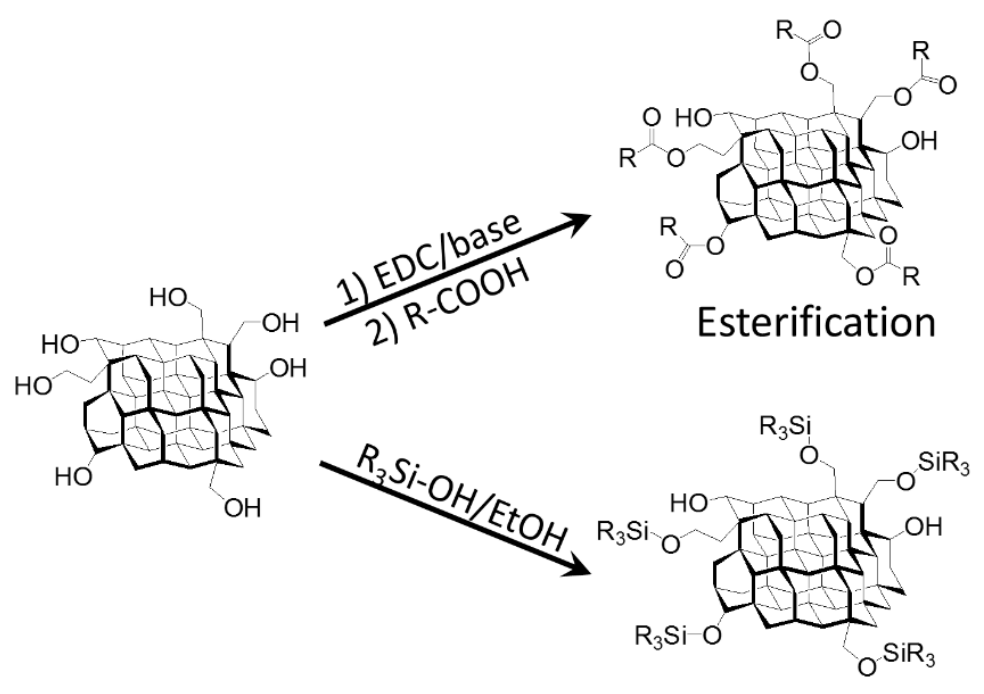

Silanization

Scheme 5. Functionalization of the hydroxyl functions of NDs $(R=$ proteins, peptides, amino-alkyl, glycidyl moieties).

Siloxane functionalization offers several advantages: it can be performed under mild conditions using alcoholic solvents and the reaction is highly versatile and tunable due to the wide variety of siloxanes commercially available. On the other hand, siloxane functionalization may suffer from hydrolysis and so crosslinking agents such as 1,2-bis (triethoxysilyl)ethane are required. Noteworthy, the reaction with siloxanes forms a uniform shell around the NDs where the thickness depends on the siloxane precursor used. ${ }^{[12]}$ Siloxane functionalization can be used to enhance the surface area of the nanoparticles and so the drug loading capacity of the materials. This approach has been successfully used to covalently anchor onto ND surface both dyes and receptors via click chemistry onto silica grafted NDs with interesting results in vitro. ${ }^{[3]}$ 
The described functionalization reactions are aimed to exploit the NDs in the biomedical field. However, there are also many other possibilities that allow to modify the NDs with different functional groups. For example, hydroxylated NDs can react via Williamson reaction with alkyl chloride, while hydrogenated NDs can be easily functionalized with alkenes after UV activation or with diazonium salt through Tour reaction. In addition, ND surface can exhibit an unexpected reactivity compared to "classic" molecules. For instance, fluorinated NDs (prepared via fluorine plasma) can easily react with amines and Grignard reagents. ${ }^{[3,23]}$ In addition, carboxylic groups present onto the ND surface can be easily converted into azido group obtaining a material readily feasible for click chemistry. ${ }^{[28]}$ For these reasons, we believe that there is still room for improvement the functionalization strategy according to the final applications.

\section{Bioconjugation}

For their stiffness, lubricity, mechanical resistance and refractivity, NDs have been successfully applied to different materials science fields. However, one of the most promising application area is certainly the biomedical. Thanks to their high biocompatibility, their intrinsic inertness, their ability to easily penetrate cell membranes and their well-established surface chemistry, NDs are explored as a powerful carrier to deliver different drugs inside target cells and to monitor intracellular processes. To facilitate the biomedical applications of NDs, it is essential to conjugate them with specific biomolecules such as peptides, proteins, nucleic acids and other bioactive molecules. Depending on the interaction between the NDs and the biomolecules, the bioconjugation can be divided into non-covalent, covalent and interlayer mediated approaches. NDs are able to complex proteins or lipophilic drugs via hydrophobic interactions (Scheme 3), whereas the surface charge of NDs enables the loading of the biomolecules through electrostatic attraction (Scheme 2). ${ }^{[29]}$ Because the non-covalent bonding is quite weak and non-specific, the biomolecules could gradually detach from ND surface under biological conditions. In the case of NDs bearing functional groups like hydroxyl and carboxylic groups, it is possible to conjugate the biomolecules to NDs through covalent bonding (e.g. ester and amide bonds) that provides stronger and more stable connection (Scheme 4). To favor the practical applications of functionalized NDs in physiological 
environments, interlayers, mostly constituted of hydrophilic polymers, ${ }^{[30-32]}$ are often incorporated between the NDs and the bioactive species to enhance the aqueous dispersibility and offer more functionalities for chemical binding. Among the different interlayers, PEG chains terminated with various functional moieties such as amino, thiol and azido groups are frequently employed to selectively bind a range of biomolecules through covalent bonds. ${ }^{[11]}$ As an alternative strategy to PEGylation, PG grafting and further derivatization of NDs have recently been developed to make the bioconjugation more convenient and economic (Scheme 6). ${ }^{[25,26]}$ The grafted PG layer on NDs has a hyperbranched topology and possesses many hydroxyl groups at the periphery, which not only significantly improve the hydrophilicity, but can be easily converted to more reactive functional groups (e.g. amino, azido and carboxylic groups), for further bioconjugation and drug loading. Up to now, a wide range of molecules including targeting peptides, ${ }^{[33]}$ chemotherapeutics ${ }^{[26,34]}$ and plasmid $D N A^{[35,36]}$ have been conjugated to the surface engineered ND-PG (Scheme 6), demonstrating preserved biological activities.

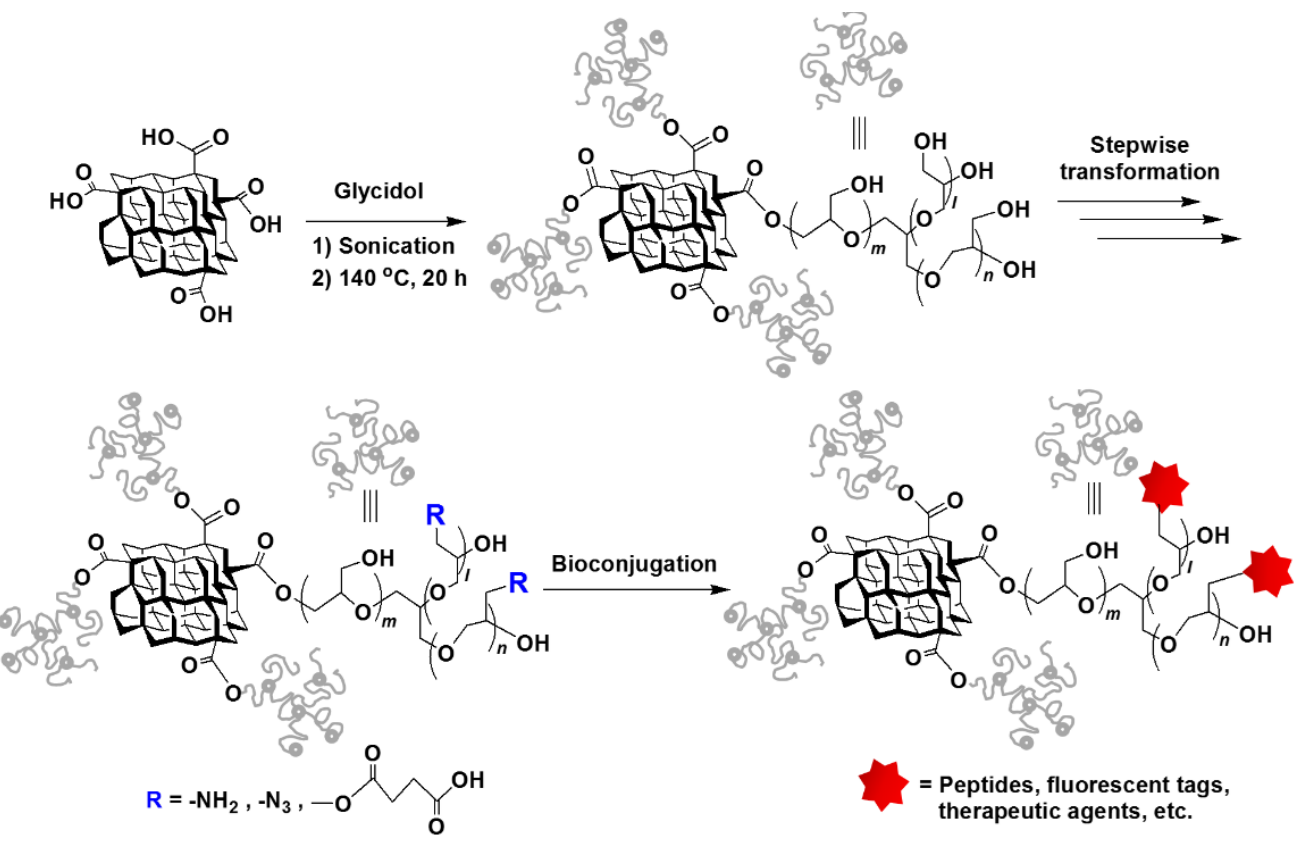

Scheme 6. Conjugation of NDs with biomolecules through the PG mediated approach. 


\section{Characterizations of NDs}

One important aspect associated to the chemistry on NDs concerns the characterization of the precursors and the final conjugates. The techniques that allow to analyze the quality of ND core and surface can be divided into two families, 1) microscopic and 2) macroscopic techniques. The former includes X-ray photoelectron (XPS) spectroscopy, scanning and transmission electronic microscopies (SEM, TEM), selective area electron diffraction (SAED), and energy dispersive X-ray spectrometry (EDX), while the latter comprises elemental analysis (EA), mass spectrometry, NMR (nuclear magnetic resonance), Raman, infrared (IR) and X-ray diffraction (XRD) spectroscopies, mechanical testing, and thermogravimetric analysis (TGA). ${ }^{[6,37]}$ The morphology and size (using TEM, SEM and XRD), $\mathrm{sp}^{2}$ carbon content (using XRD, Raman and NMR) and metal impurities (using EDX, EA, TGA and XPS) can be visualized and/or quantified. ${ }^{[6,37]}$ However, ND surface is very difficult to precisely characterize due to its complexity. Generally, IR, XPS and TGA are used to provide an estimation of the type and quantity of the organic groups present. ${ }^{[21]}$ In general, multiple techniques are required for ND full characterization. In this direction, we will benefit of a current rapid instrument innovation trend where the coupling of more spectroscopies (i.e. TGA/mass, Raman/XPS) or new implementation (i.e. surface enhanced Raman spectroscopy) are tremendously improving the quantity and quality of the information we can get from NDs. For instance, IR spectroscopy using diffuse reflectance has been used to analyze the ND local interactions with the adsorbed molecules thus helping to understand ND interaction with drugs. ${ }^{[38]}$

\section{Biomedical applications}

Owing to their high biocompatibility, their rich surface chemistry and the unique magnetooptical properties, NDs have attracted great attention in the area of biosystems including bioimaging, temperature sensing, drug delivery and tissue engineering (Fig. 3). 


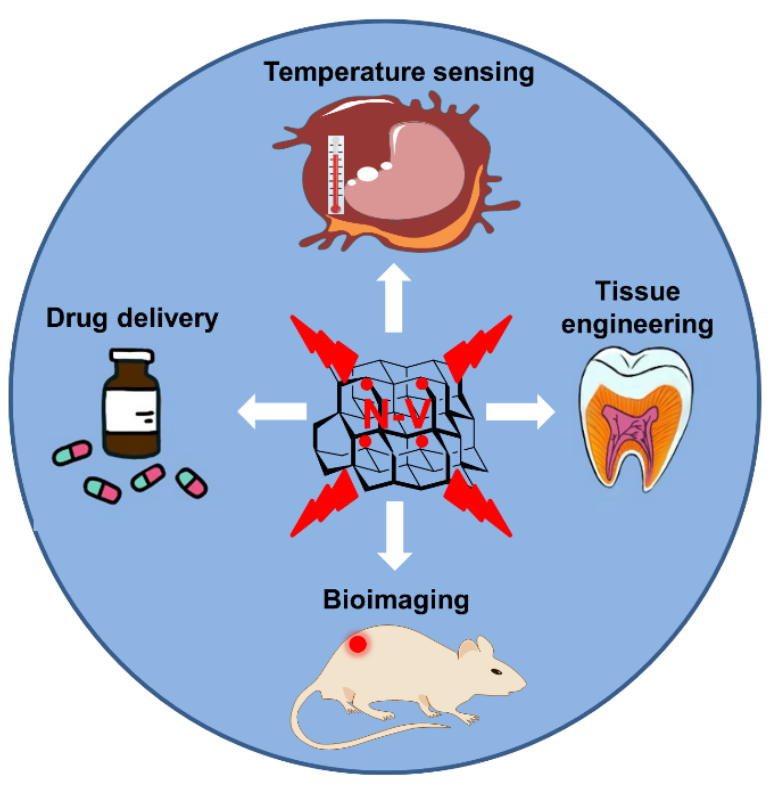

Figure 3. Biomedical applications of functionalized NDs.

NDs can incorporate a variety of color centers such as N-V centers, nickel-nitrogen complex centers, and silicon vacancy (Si-V) centers, ${ }^{[39,40]}$ which upon photo-excitation can emit bright and non-bleaching fluorescence with emission wavelengths ranging from visible to near-infrared region. The intrinsic luminescence makes NDs an ideal tool for fluorescence tracking and imaging. ${ }^{[41]}$ More attractively, taking advantage of the fluorescence and magneto-optical properties of negatively charged $\mathrm{N}-\mathrm{V}$ centers $\left(\mathrm{NV}^{-}\right)$, it is feasible to achieve background-free fluorescence imaging through different approaches. One approach relies on the fact that the fluorescence lifetime $(\tau)$ of $\mathrm{NV}^{-}(\tau \approx$ $25 \mathrm{~ns}$ ) in NDs is much longer than that of autofluorescence from biological samples ( $\tau \approx$ 1-4 ns). Therefore, the background fluorescence can be eliminated by time-gating technique. ${ }^{[42]}$ The other approach is based on the unparalleled magneto-optical property of $\mathrm{NV}^{-}$whose fluorescence intensity is related to the ground state spin configuration, which can be manipulated by electron spin magnetic resonance. According to this characteristics, either microwave radiation ${ }^{[43]}$ or a modulated magnetic field ${ }^{[44]}$ can be employed to induce a change in fluorescence intensity of $\mathrm{NV}^{-}$, whereas autofluorescence of the biomolecules have no response to the external electromagnetic fields. As a consequence, the signal-to-noise ratio of fluorescence imaging in cells and tissues is significantly increased. For instance, microwave radiation was used to image NDs in living 
C. elegans using a selective imaging protocol (SIP) based on recording the fluorescence intensity varying the electron spin magnetic resonance (Fig. 4).

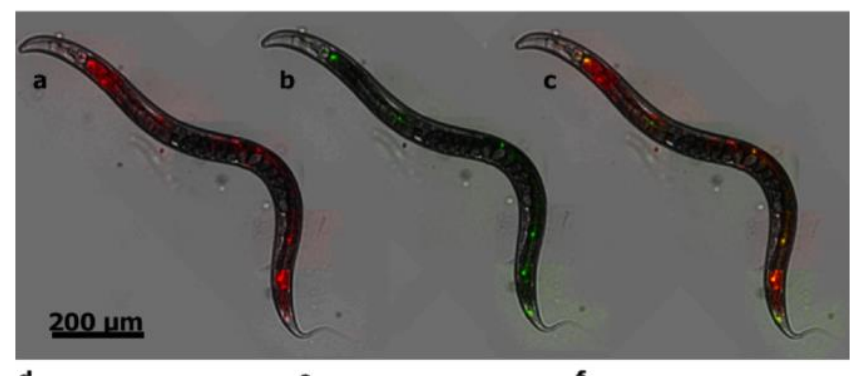

Figure 4. Imaging of NDs inside C. elegans after feeding. A conventional fluorescence image obtained without SIP is shown in red (a). An image obtained by SIP is shown in green (b). The merged image (c) reveals the fluorescence of NDs in yellow. Adapted with permission from ref. [43]. Copyright 2012, American Chemical Society

Apart from fluorescence imaging, NDs have also been intensely investigated as contrast agents in magnetic resonance imaging (MRI). Immobilization of paramagnetic gadolinium complexes on the surface of NDs can efficiently increase longitudinal relaxivity, resulting in enhanced $T_{1}$-weighted MRI. ${ }^{[45,46]}$ Similarly, Mn functionalized NDs have been used as MRI contrast agents to monitor liver tumors in vivo in a mouse model, up to 120 min postinjection (Fig. 5). ${ }^{[47]}$

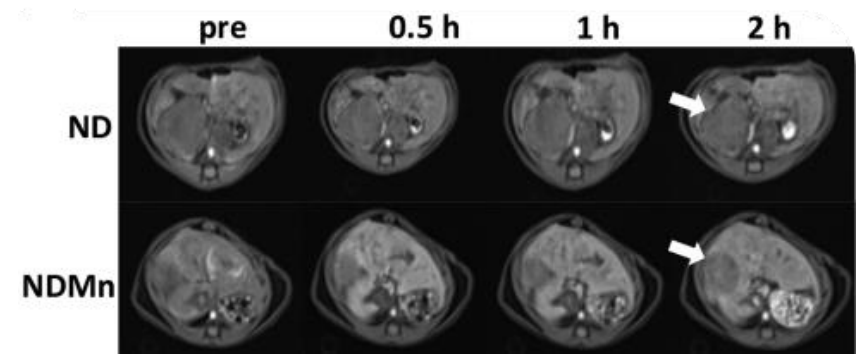

Figure 5. In vivo $T_{1}$-weighted images using NDs and NDs functionalized with $\mathrm{Mn}$ (NDMn) as contrast enhanced MR agents at different time-points. Arrows point the tumor tissues. Adapted with permission from ref. [47]. Copyright 2017, Elsevier. 
Recently, dynamic nuclear polarization through contact with electronic spins has emerged as a promising approach to enhance the sensitivity of NMR. It has found that both N-V centers ${ }^{[48]}$ and ${ }^{13} \mathrm{C}$ spins ${ }^{[49]}$ in the ND core can be hyperpolarized to act as probes in NMR imaging. ${ }^{[50]}$ The intrinsic electron spins on the surface of NDs have shown the capability of hyperpolarizing the ${ }^{1} \mathrm{H}$ nuclear spins of adsorbed liquid compounds using microwaves at room temperature, providing another possibility for ND-enhanced NMR imaging.

A precise measurement of the temperature in living systems is very challenging but of great importance for the biological and medical research. Since the fluorescence properties of $\mathrm{N}-\mathrm{V}$ centers are very sensitive to the local temperature, a ND-based nanoscale thermometry with sub-degree temperature resolution and high spatial resolution has been developed through coherent manipulation of the electronic spin associated with N-V centers. ${ }^{[51]}$ More attractively, temperature-gradient mapping at the subcellular level was realized by internalizing both NDs and gold nanoparticles into a living cell.

NDs are among the most promising nanocarriers for delivery of chemotherapeutics because of their low toxicity and large surface area. The drug loading onto the surface of NDs can be achieved by non-covalent and covalent methods. As a representative example of non-covalent loading, Ho and coworkers have developed a simple and efficient approach to complex anticancerous doxorubicin (DOX) with NDs by adding $\mathrm{NaCl}$ or $\mathrm{NaOH}$ into the aqueous dispersion. ${ }^{[52,53]}$ The sustained release of DOX from ND-DOX composites overcame chemoresistance and significantly increases cell apoptosis, and suppressed tumor growth more efficiently than conventional DOX treatment in mouse models (Fig. 6). 

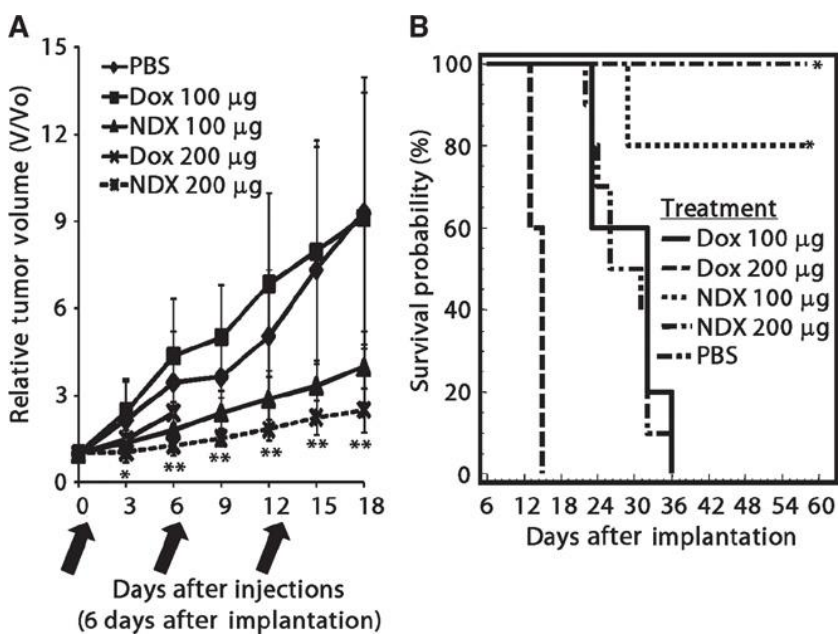

Figure 6. ND delivery of DOX inhibits tumor growth in a murine mammary carcinoma model. A) 4T1 cells were injected into the mammary gland of BALB/c virgin female mice. Mice were treated with PBS, DOX $(100 \mu \mathrm{g})$, ND-DOX (NDX) (100 $\mu \mathrm{g}$ of DOX equivalent), Dox $(200 \mu \mathrm{g})$, or NDX (200 $\mu \mathrm{g}$ of DOX equivalent) by tail vein injection. Black arrows denote injection days. B) Survival plot of treated 4T1 mice. NDX treatments showed the highest therapeutical benefits both in tumor growth and survival. Adapted from [53]. Copyright 2011, American Association for the Advancement of Science.

Through rational surface engineering, DOX was also covalently immobilized on PG grafted NDs via hydrazone bonding, which can be cleaved in mild acidic environments such as in the lysosomes of cells. ${ }^{[54]}$ Using PG as an interlayer, not only facilitates the covalent attachment of DOX, but also provides a platform to impart NDs with multiple functionalities including stealth effect to evade non-specific cell uptake and conjugation with targeting peptide. In addition to chemotherapeutics, genes and proteins can be also loaded and delivered into cells using different surface-modified NDs as carriers.

The remarkable biocompatibility and the exceptional mechanical properties of NDs make them promising candidates for applications in tissue engineering. The monolayer of NDs can act as an ideal platform for neuronal growth. It was observed that the neuron growth on ND-coated substrates show properties similar to those cultured on standard proteincoated substrates. ${ }^{[55]}$ Biodegradable poly(L-lactic acid) blended with only $10 \mathrm{wt} \%$ of octadecylamine-modified NDs displayed no negative effects on proliferation of murine 
osteoblast and a remarkable increase in hardness and Young's modulus even close to those of human cortical bone, enabling the application of the nanocomposites in bone regeneration. ${ }^{[56]}$ Recently, the first in-human administration of NDs for dental treatment has been reported by $\mathrm{Ho}$ and collaborators. ${ }^{[57,58]}$ A ND-embedded dental gutta-percha ${ }^{\mathrm{TM}}$ (GP is a natural polymer used to fill teeth canal), showing enhanced mechanical properties was utilized as a filler to repair root canal sites and prevent infections (Fig. 7).

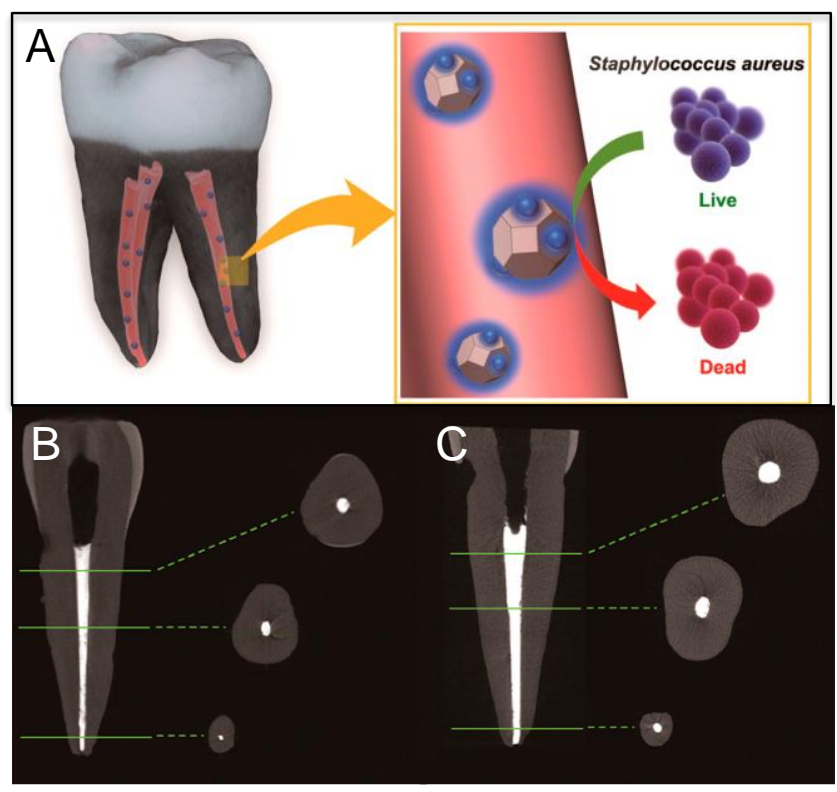

Figure 7. NDGP prevents bacterial contamination after root canal therapy due to the antimicrobial properties of both ND and amoxicillin. A) Application of NDGP embedded with ND-amoxicillin conjugates. MicroCT $(\mu \mathrm{CT})$ images of patient-derived teeth samples obturated with unmodified GP (B) and NDGP (C). Adapted with permission from [58]. Copyright 2014 American Chemical Society.

\section{Advantages and limitations of NDs}

NDs show different advantages over other nanomaterials (Table 1). First of all, they inherit many features from bulk diamond such as optical refraction and transparency, chemical inertness, exceptional hardness and mechanical strength. The intrinsic fluorescence of NDs originating from various color centers has high photostability. In particular, the unique magneto-optical properties of $\mathrm{N}-\mathrm{V}$ centers gives rise to optically detected magnetic 
resonance (ODMR) effect, which has not been found in other nanomaterials yet. In terms of biological properties, NDs show non-toxicity and excellent biocompatibility, and can be conjugated with various biomolecules. These incomparable advantages make NDs a robust tool in a broad spectrum of applications, especially in the biomedical fields.

Despite the advantages mentioned above, NDs also have their own limitations when applied. New technologies have the responsibility to solve more problems than they create. In case of NDs, they have been already commercialized for different applications such as abrasives and lubricants. Besides, considering the biomedical field different questions needs to find an answer. For instance, NDs comprising $\mathrm{sp}^{3}$ carbons are considered "biocompatible by inertness". Indeed, due to their low reactivity in biological environments, NDs are overall considered safe nanomaterials. However, this high stability even compared to other $\mathrm{sp}^{2}$ carbon nanomaterials ${ }^{[59]}$ may affect their biodegradability in the body or into the environment. ${ }^{[60]}$ It is worth to note that there are no study on biodegradability of NDs reported in the literature yet. Additionally, NDs, if not degraded, need to be excreted from the body to avoid bioaccumulation and potential longterm safety issues. Different in vivo studies have been performed to answer these questions. ${ }^{[5]}$ Owing to their high surface energy and large specific surface area, NDs are prone to aggregate and adsorb many proteins following intravenous administration, which may alter their biological performance. However, coronation and aggregation can be modulated via surface functionalization. ${ }^{[3]}$ In the case of in vivo fluorescence imaging, the number of luminescent centers in single NDs are very limited, leading to insufficient emission intensity that hardly penetrates skins and tissues. In addition, to create N-V centers in NDs, irradiation by high-energy electron/helium ion beams and subsequent high-temperature annealing are required, which largely increase the cost of fluorescent NDs and inevitably destroy the intrinsic surface chemistry. Last but not least, increasing the colloidal stability of fluorescent NDs to meet the requirements of in vivo studies still remains challenging. For these reasons, different groups are developing strategies to prepare highly doped NDs already at the synthetic step.

Table 1. Pros and cons of different carbon nanomaterials used in biomedicine. 


\begin{tabular}{|c|c|c|}
\hline $\begin{array}{l}\text { Type of carbon } \\
\text { nanomaterials }\end{array}$ & Pros & Cons \\
\hline Nanodiamonds & $\begin{array}{l}\text { Chemically inert, good mechanical } \\
\text { properties, intrinsic fluorescence, dense } \\
\text { functional groups, ODMR effect for } \\
\text { biosensing }\end{array}$ & $\begin{array}{l}\text { Unknown degradability, insulator, irregular } \\
\text { shape }\end{array}$ \\
\hline Fullerenes ${ }^{[61]}$ & $\begin{array}{l}\text { Defined molecular structure, controlled } \\
\text { chemical functionalization, radical } \\
\text { scavenging activity }\end{array}$ & $\begin{array}{l}\text { Low degree of functionalization, } \\
\text { hydrophobic, small size }\end{array}$ \\
\hline $\begin{array}{l}\text { Carbon } \\
\text { nanotubes }{ }^{[62]}\end{array}$ & $\begin{array}{l}\text { Good mechanical properties, size tailoring, } \\
\text { good cell uptake, photothermal properties, } \\
\text { near-infrared fluorescence }\end{array}$ & $\begin{array}{l}\text { Hydrophobic, size dependent toxicity, risk } \\
\text { of organ accumulation, inflammatory } \\
\text { potential }\end{array}$ \\
\hline Graphene $e^{[63]}$ & $\begin{array}{l}\text { Good mechanical properties, size tailoring, } \\
\text { facile preparation, photothermal properties }\end{array}$ & $\begin{array}{l}\text { Hydrophobic, low functionalization level, no } \\
\text { fluorescence }\end{array}$ \\
\hline Graphene oxide ${ }^{[63]}$ & $\begin{array}{l}\text { Size tailoring, very high water dispersibility, } \\
\text { facile preparation, photothermal properties, } \\
\text { dense functional groups }\end{array}$ & $\begin{array}{c}\text { Batch to batch variability, wide size } \\
\text { distribution, low fluorescence, inflammatory } \\
\text { potential }\end{array}$ \\
\hline
\end{tabular}

\section{Summary and Perspective}

NDs are a family of material constituted of a well-defined core and a functional surface. The ND core is composed exclusively of $\mathrm{sp}^{3}$ carbon lattice covalently bound each other, making them one of the hardest and most stable material. Moreover, the lattice can also give the opportunity to make color centers, not only emitting fluorescence, but also susceptive to magnetic field. In contrast to the robust nature of the core, the surface of NDs is more dynamic. Indeed, from a material chemistry point of view, the NDs can be treated as organic compounds. The well-established surface chemistry developed in the last years has enormously widened their applications in different technological fields. As mentioned above, the functional groups on ND surface can be controlled through oxidation, reduction and then further functionalized with organic moiety covalently or noncovalently for various purposes.

We would like to encourage the scientists working with NDs to design future systems taking into account the synergistic properties of the ND core and surface. For mechanical applications, the "small" and "hard" properties of NDs have been utilized as abrasives, but not so much attention has been paid to the surface. The chemical modification with appropriate functional groups can impart more functions to NDs, for example in the drug delivery, in vitro or in vivo sensing or in tissue engineering. To date, great effort has been currently invested to push forward these materials in clinics. ${ }^{[64]}$ In addition, the surface modification of NDs can also influence their core properties including fluorescence and 
even electron spin properties of $\mathrm{N}-\mathrm{V}$ centers. For example, surface oxidation is often employed to generate oxygen-containing functional groups on NDs. This process is also necessary to remove blackish graphitic layers on fluorescent NDs, effectively enhancing the fluorescence intensity upon photoexcitation. ${ }^{[26]}$ On the other hand, a recent research unveils that surface oxidation of NDs is capable of extending the transverse spincoherence time $\left(T_{2}\right)$ of $\mathrm{N}-\mathrm{V}$ centers, which dictates the spin-memory time in quantum devices and the sensitivity of quantum. ${ }^{[65]}$ Taking into account the different preparation methods and the broad range of surface modifications that can be applied to NDs, we expect that such materials will find more and more uses in materials science and biomedicine. Both core and surface are expected to be synergized in future applications of NDs.

\section{Acknowledgments}

The authors gratefully acknowledge the financial support from the Agence Nationale de la Recherche (ANR) through the LabEx project Chemistry of Complex Systems (ANR-10LABX-0026_CSC). This work was partly supported by the Centre National de la

Recherche Scientifique (CNRS), the International Center for Frontier Research in Chemistry (icFRC), and JSPS KAKENHI Grant Number 17H02738. AB and LZ are indebted to Kyoto University for an Invited Professorship in 2019 and 2016, respectively.

\section{References}

[1] K. Larsson, Y. Tian, Carbon 2018, 134, 244-254.

[2] R. Namdar, S. Nafisi, Drug Discov. Today 2018, 23, 1152-1158.

[3] J. Whitlow, S. Pacelli, A. Paul, J. Control. Release 2017, 261, 62-86.

[4] A. Nagl, S. R. Hemelaar, R. Schirhagl, Anal. Bioanal. Chem. 2015, 407, 75217536.

[5] M. Chipaux, K. J. van der Laan, S. R. Hemelaar, M. Hasani, T. Zheng, R. Schirhagl, Small 2018, 14, 1704263.

[6] K. Turcheniuk, V. N. Mochalin, Nanotechnology 2017, 28, 252001. 
[7] L. Cao, C. Gao, H. Sun, G. Zou, Z. Zhang, X. Zhang, M. He, M. Zhang, Y. Li, J. Zhang, et al., Carbon 2001, 39, 311-314.

[8] M. N. Regueiro, P. Monceau, J.-L. Hodeau, Nature 1992, 355, 237-239.

[9] O. A. Shenderova, G. E. McGuire, Biointerphases 2015, 10, 030802.

[10] L.-J. Su, C.-Y. Fang, Y.-T. Chang, K.-M. Chen, Y.-C. Yu, J.-H. Hsu, H.-C. Chang, Nanotechnology 2013, 24, 315702.

[11] W. W.-W. Hsiao, Y. Y. Hui, P.-C. Tsai, H.-C. Chang, Acc. Chem. Res. 2016, 49, 400-407.

[12] J. Neburkova, J. Vavra, P. Cigler, Curr. Opin. Solid State Mater. Sci. 2017, 21, 43-53.

[13] V. N. Mochalin, O. Shenderova, D. Ho, Y. Gogotsi, Nat. Nanotechnol. 2012, 7, 11-23.

[14] O. Shenderova, N. Nunn, Nanodiamonds 2017, 25-56.

[15] V. Y. Dolmatov, Russ. Chem. Rev. 2007, 76, 339-360.

[16] A. M. Schrand, S. A. C. Hens, O. A. Shenderova, Crit. Rev. Solid State Mater. Sci. 2009, 34, 18-74.

[17] A. Krüger, F. Kataoka, M. Ozawa, T. Fujino, Y. Suzuki, A. E. Aleksenskii, A. Y. Vul', E. Ōsawa, Carbon 2005, 43, 1722-1730.

[18] A. Pentecost, S. Gour, V. Mochalin, I. Knoke, Y. Gogotsi, ACS Appl. Mater. Interfaces 2010, 2, 3289-3294.

[19] A. Gismondi, G. Reina, S. Orlanducci, F. Mizzoni, S. Gay, M. L. Terranova, A. Canini, Biomaterials 2015, 38, 22-35

[20] C. Shuai, Y. Li, G. Wang, W. Yang, S. Peng, P. Feng, Int. J. Biol. Macromol. 2019, 126, 1116-1124.

[21] G. Reina, A. Gismondi, R. Carcione, V. Nanni, C. Peruzzi, M. Angjellari, N. D. Q. Chau, A. Canini, M. L. Terranova, E. Tamburri, Appl. Surf. Sci. 2019, 470, 744754.

[22] P. Wang, W. Su, X. Ding, Diam. Relat. Mater. 2018, 88, 43-50.

[23] H. Tinwala, S. Wairkar, Mater. Sci. Eng. C 2019, 97, 913-931.

[24] Y. Liu, Z. Gu, J. L. Margrave, V. N. Khabashesku, Chem. Mater. 2004, 16, 39243930. 
[25] L. Zhao, T. Takimoto, M. Ito, N. Kitagawa, T. Kimura, N. Komatsu, Angew. Chemie Int. Ed. 2011, 50, 1388-1392.

[26] L. Zhao, Y.-H. Xu, H. Qin, S. Abe, T. Akasaka, T. Chano, F. Watari, T. Kimura, N. Komatsu, X. Chen, Adv. Funct. Mater. 2014, 24, 5348-5357.

[27] D. Terada, S. Sotoma, Y. Harada, R. Igarashi, M. Shirakawa, Bioconjug. Chem. 2018, 29, 2786-2792.

[28] Z. C. Kennedy, C. A. Barrett, M. G. Warner, Langmuir 2017, 33, 2790-2798.

[29] Y.-K. Tzeng, O. Faklaris, B.-M. Chang, Y. Kuo, J.-H. Hsu, H.-C. Chang, Angew. Chemie Int. Ed. 2011, 50, 2262-2265.

[30] L.-C. L. Huang, H.-C. Chang, Langmuir 2004, 20, 5879-5884.

[31] T. Takimoto, T. Chano, S. Shimizu, H. Okabe, M. Ito, M. Morita, T. Kimura, T. Inubushi, N. Komatsu, Chem. Mater. 2010, 22, 3462-3471.

[32] B.-M. Chang, H.-H. Lin, L.-J. Su, W.-D. Lin, R.-J. Lin, Y.-K. Tzeng, R. T. Lee, Y. C. Lee, A. L. Yu, H.-C. Chang, Adv. Funct. Mater. 2013, 23, 5737-5745.

[33] T.-F. Li, K. Li, Q. Zhang, C. Wang, Y. Yue, Z. Chen, S.-J. Yuan, X. Liu, Y. Wen, M. Han, et al., Biomaterials 2018, 181, 35-52.

[34] L. Zhao, H. Yang, T. Amano, H. Qin, L. Zheng, A. Takahashi, S. Zhao, I. Tooyama, T. Murakami, N. Komatsu, J. Mater. Chem. B 2016, 4, 7741-7748.

[35] L. Zhao, Y. Nakae, H. Qin, T. Ito, T. Kimura, H. Kojima, L. Chan, N. Komatsu, Beilstein J. Org. Chem. 2014, 10, 707-13.

[36] X. Li, L. Zhao, Q. Liang, J. Ye, N. Komatsu, Q. Zhang, W. Gao, M. Xu, X. Chen, J. Biomed. Nanotechnol. 2017, 13, 280-289.

[37] A. M. Panich, Crit. Rev. Solid State Mater. Sci. 2012, 37, 276-303.

[38] T. Petit, L. Puskar, Diam. Relat. Mater. 2018, 89, 52-66.

[39] H.-C. Chang, W. W.-W. Hsiao, M.-C. Su, Fluorescent Nanodiamonds, Wiley 2018.

[40] M. H. Alkahtani, F. Alghannam, L. Jiang, A. Almethen, A. A. Rampersaud, R. Brick, C. L. Gomes, M. O. Scully, P. R. Hemmer, Nanophotonics 2018, 7, 14231453.

[41] C.-C. Fu, H.-Y. Lee, K. Chen, T.-S. Lim, H.-Y. Wu, P.-K. Lin, P.-K. Wei, P.-H. Tsao, H.-C. Chang, W. Fann, Proc. Natl. Acad. Sci. U. S. A. 2007, 104, 727-32.

[42] T.-J. Wu, Y.-K. Tzeng, W.-W. Chang, C.-A. Cheng, Y. Kuo, C.-H. Chien, H.-C. 
Chang, J. Yu, Nat. Nanotechnol. 2013, 8, 682-689.

[43] R. Igarashi, Y. Yoshinari, H. Yokota, T. Sugi, F. Sugihara, K. Ikeda, H. Sumiya, S. Tsuji, I. Mori, H. Tochio, et al., Nano Lett. 2012, 12, 5726-5732.

[44] R. Chapman, T. Plakhoitnik, Opt. Lett. 2013, 38, 1847.

[45] L. M. Manus, D. J. Mastarone, E. A. Waters, X.-Q. Zhang, E. A. Schultz-Sikma, K. W. MacRenaris, D. Ho, T. J. Meade, Nano Lett. 2010, 10, 484-489.

[46] L. Zhao, A. Shiino, H. Qin, T. Kimura, N. Komatsu, J. Nanosci. Nanotechnol. 2015, 15, 1076-1082.

[47] W. Hou, T. B. Toh, L. N. Abdullah, T. W. Z. Yvonne, K. J. Lee, I. Guenther, E. K.H. Chow, Nanomedicine 2017, 13, 783-793.

[48] A. Ajoy, K. Liu, R. Nazaryan, X. Lv, P. R. Zangara, B. Safvati, G. Wang, D. Arnold, G. Li, A. Lin, et al., Sci. Adv. 2018, 4, eaar5492.

[49] E. Rej, T. Gaebel, T. Boele, D. E. J. Waddington, D. J. Reilly, Nat. Commun. 2015, 6, 8459.

[50] E. Rej, T. Gaebel, D. E. J. Waddington, D. J. Reilly, J. Am. Chem. Soc. 2017, 139, 193-199.

[51] G. Kucsko, P. C. Maurer, N. Y. Yao, M. Kubo, H. J. Noh, P. K. Lo, H. Park, M. D. Lukin, Nature 2013, 500, 54-58.

[52] H. Huang, E. Pierstorff, E. Osawa, D. Ho, Nano Lett. 2007, 7, 3305-3314.

[53] E. K. Chow, X.-Q. Zhang, M. Chen, R. Lam, E. Robinson, H. Huang, D. Schaffer, E. Osawa, A. Goga, D. Ho, Sci. Transl. Med. 2011, 3, 73ra21.

[54] L. Zhao, Y.-H. Xu, T. Akasaka, S. Abe, N. Komatsu, F. Watari, X. Chen, Biomaterials 2014, 35, 5393-5406.

[55] A. Thalhammer, R. J. Edgington, L. A. Cingolani, R. Schoepfer, R. B. Jackman, Biomaterials 2010, 31, 2097-2104.

[56] Q. Zhang, V. N. Mochalin, I. Neitzel, I. Y. Knoke, J. Han, C. A. Klug, J. G. Zhou, P. I. Lelkes, Y. Gogotsi, Biomaterials 2011, 32, 87-94.

[57] D.-K. Lee, T. Kee, Z. Liang, D. Hsiou, D. Miya, B. Wu, E. Osawa, E. K.-H. Chow, E. C. Sung, M. K. Kang, et al., Proc. Natl. Acad. Sci. U. S. A. 2017, 114, E9445E9454.

[58] D.-K. Lee, S. V. Kim, A. N. Limansubroto, A. Yen, A. Soundia, C.-Y. Wang, W. 
Shi, C. Hong, S. Tetradis, Y. Kim, et al., ACS Nano 2015, 9, 11490-11501.

[59] A. Bianco, K. Kostarelos, M. Prato, Chem. Commun. 2011, 47, 10182.

[60] R. Kurapati, S. P. Mukherjee, C. Martín, G. Bepete, E. Vázquez, A. Pénicaud, B. Fadeel, A. Bianco, Angew. Chemie Int. Ed. 2018, 57, 11722-11727.

[61] A. Montellano, T. Da Ros, A. Bianco, M. Prato Nanoscale 2011, 3, 4035-4041.

[62] K. Kostarelos, K.; Bianco, A.; Prato, M. Nat. Nanotech. 2009, 4, 627-633

[63] G. Reina, J.M. González-Domínguez, A. Criado, E. Vázquez, A. Bianco, M. Prato, Chem. Soc. Rev. 2017, 46, 4400-4416.

[64] L. Moore, J. Yang, T. T. H. Lan, E. Osawa, D.-K. Lee, W. D. Johnson, J. Xi, E. K.H. Chow, D. Ho, ACS Nano 2016, 10, 7385-7400.

[65] R. Tsukahara, M. Fujiwara, Y. Sera, Y. Nishimura, Y. Sugai, C. Jentgens, Y. Teki, H. Hashimoto, S. Shikata, ACS Appl. Nano Mater. 2019, doi:10.1021/acsanm.9b00614 
